\title{
A Condensation Theory of Meteoric Matter and its Cosmological Significance
}

\section{By Prof. Bertil Lindblad, Director of Stockholm Observatory}

$I^{N}$ connexion with a theory on the constitution recently directed attention ${ }^{1}$ to the significance of the great difference in temperature between the interstellar gas and solid interstellar particles as an explanation of the origin and growth of meteoric particles. If we assume with Sir Arthur Eddington ${ }^{2}$ a temperature of $10,000^{\circ}$ for the interstellar gas and, on account of the low energy density, a temperature of about $3^{\circ}$ for solid particles, the latter must be assumed to grow by the condensation of sublimed matter on their surface. This conclusion is in accordance with the conclusions drawn by I. Langmuir ${ }^{3}$ concerning the nature of the process of condensation of metallic vapours on solids. In the present case, the energy of impact of atoms on the surface of the particle will be rapidly radiated into space, or perhaps to some small extent transformed into sub-atomic energy, so that the particle remains cold. We assume that the interstellar gas actually contains all the elements in about the proportions formed in the earth's crust and in the sun, and that the apparent predominance of calcium and sodium is due to the easy accessibility of very strong spectral lines due to these elements, namely, the $H$ and $K$ lines and the $D$ line. For atomic weight 50, the temperature $10,000^{\circ}$ gives a mean speed of the atoms of $2 \mathrm{~km}$. per sec., and assuming a density of 5 for the solid particles formed, we readily obtain the formula

$$
m=10^{36}(\rho t)^{3},
$$

for the mass $m$ of the particle in grams after the time $t$ since the formation of an 'infinitesimal' nucleus expressed in years. For the density $\rho$ of the interstellar gas we may adopt Gerasimovic and Struve's value, $10^{-26} \mathrm{gm}$. per $\mathrm{cm}^{3}$. In the time $t=10^{\circ}$ years we then get particles of the size $10^{-15} \mathrm{gm}$., which is the order of magnitude of the particles in the obscuring clouds of the Milky Way according to the recent results derived by C. Schalén ${ }^{5}$ from an investigation of the absorption effects in the photographic spectrum of early type stars in various Milky Way regions and an application of Mie's theory of the absorption of light by colloidal particles. It is remarkable that the mean density derived by Schalén from the number of particles per cubic centimetre agrees well with Gerasimovic and Struve's value for the interstellar gas. We may further remark here that the time of $10^{\circ}$ years agrees with the average order of magnitude of the age of meteorites derived by an analysis of radioactive material.
The result obtained for the small interstellar particles encourages us to apply our formula to the formation of meteoric material in somewhat denser regions in space as well. Before leaving the small obscuring particles, we may add, however, that these particles will adopt motions corresponding to the mean motion of the interstellar gas, which we know follows closely the rotation of the stellar stratum around the centre of the stellar system situated in the direction of the constellation Sagittarius. (The distance to the centre is estimated to be 10,000 parsecs, or about 30,000 light-years. The period of rotation at the position of the sun in the system is nearly $2 \times 10^{8}$ years.) The consequence will be that the particles will get only small velocities relative to the circular motions, and will show a strong galactic concentration, presumably much stronger than the galactic concentration of the interstellar gas itself. This result is quite in accordance with the empirical data regarding the region of obscuration in our stellar system.

For an accumulation of material through gravitational forces to be possible in the case of an angular velocity of rotation corresponding to the period of revolution just mentioned, we should have a minimum density of about $10^{-22} \mathrm{gm}$. per $\mathrm{cm} .^{3}$. In $10^{9}$ years we get with such a density particles of $10^{-3} \mathrm{gm}$., which seems to be the minimum mass of observed meteors. We assume here throughout a temperature of about $10,000^{\circ}$ for the gas and a temperature near zero (absolute) in the case of the particles. Even rather large changes in the temperature of the gas, however, will not affect the order of magnitude of $\boldsymbol{m}$.

If we assume the sun and the planetary system to have evolved from a gaseous nebula, stretching at a certain time over the orbits of Neptune and Pluto with a reasonable concentration towards the central nucleus, we might estimate the density in the outer regions of such a nebula to be something like $10^{-15} \mathrm{gm}$. per $\mathrm{cm} .^{3}$. We should then expect particles of $10^{18} \mathrm{gm}$., that is, one million million tons, to develop in $10^{9}$ years. This is about the mass of one of the smallest asteroids. We do not suggest, however, that bodies of this size will go on condensing in this way undisturbed. We must expect particles of various size to combine occasionally to form still larger formations. On the other hand, especially in the denser regions near the central nucleus, the particles will run a great risk of being volatilised by violent encounters, and with increasing energy-density the temperature of particles will be too high to admit condensation. 
Further, inside Roche's limit around the centre no formation of large particles is possible. Therefore there will probably be an optimum region for the formation of large bodies in the solar system, and we may assume this region to occur around the orbits of the giant planets Jupiter and Saturn. We may mention here the well-known fact that the planet Jupiter contributes about 60 per cent of the total angular momentum of the solar system.

The objection by H. Jeffreys' to the 'planetesimal theory' of Chamberlain and Moulton that the planetesimals are likely to volatilise entirely before contributing essentially to the formation of larger bodies of small orbital eccentricities and inclinations does not apply generally in our case, since the condensed particles are likely to possess from the beginning only small velocities relative to the circular orbits in the plane of rotation of the nebulous mass. In the extended solar nebula, viscosity will have produced at least roughly regular conditions, and because the pressure gradient is not likely to contribute much to support the gaseous matter against gravity, the mean motion of the gas, and hence the motion of the condensed particles, is likely to follow fairly closely the circular motion. I have further shown as a general theorem ${ }^{7}$ that mild encounters leading to a dissipation of kinetic energy of the particles will, in the mean, lead to a concentration of the particles towards circular orbits in the plane of rotation. Still, owing to the large free surface per unit mass of the small particles, a very great number of these corpuscles will actually be evaporated by encounters; but some particles which succeed in growing without serious interruption will increase in size by accumulation of smaller particles at a rate probably exceeding considerably that of our condensation formula given above. It appears, therefore, that the net result of the condensation process will ultimately be a rapid growth of a comparatively small number of large bodies. We of course at once identify these bodies with the planets and their satellites.

We may ask, incidentally, if there are in the universe types of objects which in any way resemble the primordial solar system as depicted above. In actual fact, it is not unreasonable to assume that certain stars of early type with nebulous envelopes showing the reflected continuous spectra of the stars involved, which are not seldom observed in the Milky Way, constitute systems of a similar nature ; that is, they consist of a stellar core inside a thin gaseous envelope which in the outer regions passes over into a meteoric cloud. When, however, the central nucleus reaches a very high temperature, there must be a strong radiation pressure on particles of a certain size $e^{8}$. In such a case we should expect not only an out- going stream of small particles, for which radiation pressure increases with increasing size, but also an incoming stream of larger particles, for which the radiation pressure decreases with increasing diameter. It is possible, further, that larger particles have formed at some time before the nucleus acquired by contraction a very high surface temperature. Modern evidence ${ }^{9}$ seems actually to indicate reflection by fairly large particles in the nebulous envelope. We may assume that the types of objects in our stellar system which show the strongest galactic concentration have actually formed in the 'present phase' of the stellar system, that is, during the time in which the stellar system has possessed its present mass, angular momentum and invariable plane. The minimum amount of time passed in this phase may perhaps be set down as $10^{10}$ years. This seems to be near the upper limit of the age of the earth's crust. If the sun, or rather the solar nebula, became differentiated out of the interstellar material in the present phase of the system, or during an earlier phase, seems therefore doubtful.

Returning to the formation of the planets from the gaseous and meteoric material of the solar nebula, in accordance with the lines of thought just outlined, we assume that the earth has been formed around a heavy core by accumulation of a great number of particles of various sizes. In addition to the considerations dealt with earlier, we may direct attention to the circumstance that, between neighbouring particles formed by condensation of atoms of very large mean free path, and by accumulation of free particles of larger size, there will be a certain additional pull due to their shielding one another with respect to the free atoms or corpuscles falling in towards their surfaces. As the accumulation may be thought to have been especially rapid at certain stages of the process, for example, in encounters with comparatively large bodies, the planet may ultimately have become heated to such an extent that at least the surface layers have been entirely in a fluid state, perhaps even incandescent. A temperature of $2,000^{\circ}$ of the radiating surface may be upheld, if material of a mean space density about $10^{-9} \mathrm{gm}$. per $\mathrm{cm} .^{3}$ falls in towards the radiating surface with a mean velocity of $10 \mathrm{~km}$. per sec. At a later stage, through loss of heat by radiation, simultaneous solidification all over the surface has taken place.

The formation of satellite systems is explained on general lines in a way analogous to the theory of formation of the planetary system itself. The fusion of particles of various sizes, leading to the formation of a massive planet, will in the first stage produce an incandescent nucleus with an extended gaseous atmosphere. The angular 
momentum due to the satellite system and the rotation of the planet taken together correspond to the angular momentum of the matter which was sufficiently near a planetary nucleus to be retained in its neighbourhood by gravitation, or in the course of time has been caught in encounters. The direction of rotation should therefore be the same as that of the system as a whole, and thus that of the planetary orbits; the few exceptions to this rule are readily explained as accidental deviations due to local conditions (the Uranus system and the satellite of Neptune) or capture in recent times (Jupiter VIII and IX, Saturn IX). The strong correlation between the angular momentum of rotation of the planet and the mass of the satellite system is readily understood. Apparently singular objects like the satellites of Mars may be understood without making the assumption that these bodies have originated directly out of the mass of the planet.

The rings of Saturn are perhaps best explained directly as small particles formed by condensation inside Roche's limit for the planet in question. The extremely flat formation is explained partly by the condensation of the particles out of a gaseous cloud, which causes the particles to follow originally nearly circular motions with low inclinations, but mainly by the influence of mild collisions between neighbouring particles, as explained by Jeffreys ${ }^{10}$ and on somewhat different lines by myself in a recent paper ${ }^{11}$.

The corpuscles of the zodiacal light have possibly been formed fairly recently ${ }^{12}$ out of the last remnants, perhaps reformed by volatilisation, of the nebulous cloud surrounding the sun.

Finally we must mention the probable status of the meteorites. Their division into, roughly, two widely different kinds, stony and iron meteorites, suggests that they can scarcely be considered exclusively as direct condensation products. We may perhaps get a general explanation of their physical nature, as well as of their motions, which obviously differ very much from the circular orbits in the invariable plane of the solar system, by assuming that they are, generally speaking, remnants of larger bodies, shattered by violent encounters in the manner which has been considered above. Like the planets, these larger bodies have therefore formed to a great extent by accumulation of smaller particles. We can then assume, in conformity with the views put forward by geophysicists ${ }^{13}$, that a certain sedimentation of the various minerals has taken place at a fairly high temperature in the bodies in question before their disruption. Those meteorites which did not originally belong to our system have probably emanated from analogous systems in other regions of the Milky Way, forming showers of particles traversing the voids of interstellar space much like the stars themselves. In regard to their origin they are, according to our point of view, largely a sort of by-product in the process of formation of planetary systems.

${ }^{1}$ Mon. Not. Roy. Ast. Soc., in press.

2 "The Internal Comstitution of the Stars" p. 371 ; 1926.

${ }^{3}$ Phys. Rev., 8, 149; 1916. Proc. U. S. Nat. Acad., 3, 141; 1917.

Astrophys. J., 69, 7 ; 1929

KK. Sven. Vetenskap., Handl., 3, 13, No. 2; 1934. (Upsala Medde lande, No. 58.)

"The Earth", p. $250 ; 1924$

Mon. Not. Roy. Ast. Soc., 94, 231 ; 1934

${ }^{8}$ Cf. Schoenberg and Jung, Astron.' Nachr., 247, 413 (1933).

${ }^{\circ}$ Cf. Struve, Elvey and Keenan, Astrophys., $J ., 77,274 ; 1934$

${ }^{10}$ Mon. Not. Roy. Ast. Soc., $7 \mathrm{r}, 91 ; 1916$.

${ }^{11}$ loc. cit.

${ }^{19}$ Cf. H. Jeffreys, loc. cit.

13 Cf. G. v. Hevesy, "Chemical Analysis by X-Rays and its Applications". (Cornell University Publ. 1932.)

\section{Deep Diving and Under-Water Rescue}

CIR ROBERT DAVIS delivered the Thomas $S_{\text {Gray Memorial Lectures for } 1934 \text { of the Royal }}$ Society of Arts, and spoke on deep diving and under-water rescue. The lectures have now been published; they form a valuable study of the development of apparatus and technique, admirably illustrated and lightened by comments and anecdotes arising out of the author's lifelong experience of the subject.

Divers have always wanted to get a little deeper, but the particular obstacle to be overcome has varied in successive generations. At first it was the matter of air supply, when the eighteenth century inventor and his victims "discovered by bitter experience that the leathern bellows, which worked so admirably when blowing an organ or smith's forge, were quite incapable of forcing air down to a diver" working at more than two or three feet deep. Later, when the introduction of metal air pumps enabled a supply to be delivered at high pressure, came the mysterious, crippling, 'diver's palsy' which we now call compressed air illness. Later still, when physiologists had elucidated the cause of this trouble and devised methods of slow decompression to avert it, came the economic difficulty that at depths of thirty fathoms and upwards, so much of the diver's time under water had to be used in decompression that only a fraction remained available for useful and paying work. Now the invention of the Davis Submerged Decompression Chamber (see Nature, August 29, 1931) has eased this situation and made salvage work in the rubber dress a practical procedure up to 300 feet depth; and there for the moment we rest.

The deep diver of to-day receives a measured supply of clean air from steam compressors. An 